\title{
Value-Based Design for Gamifying Daily Activities
}

\author{
Mizuki Sakamoto, Tatsuo Nakajima, and Todorka Alexandrova \\ Department of Computer Science and Engineering, \\ Waseda University \\ \{mizuki, tatsuo, toty\}@dcl.cs.waseda.ac.jp
}

\begin{abstract}
Computing technologies allow us to gamify our daily activities by embedding computers in our environments. In this paper, we propose a valuebased gamification framework for increasing intrinsic motivation in our daily life. We introduce five values and a conceptual framework based on these values. Then, as an example we show how the values are used in Augmented Trading Card Game during its design.
\end{abstract}

\section{Introduction}

Daily digital objects are becoming more and more usual and widely sold as commodities. For example, recently televisions developed in Japan have become cheaper and cheaper despite of their excellent product quality and rich functionality. However, on the contrary new furniture and fashion goods attract us every year and they do not become commodities that are sold at cheaper prices with the time. The reason for this is the fact that they offer additional values to users. Especially, the prices for such products are kept high if the products offer the sense of rarity. The fact that the product quality does not become the main value for many of us to buy the product shows that we need to consider another way to design daily digital objects. Digital technologies are effective to make digital objects usual commodities and as a consequence to decrease their prices, but they are also effective to add more values to products by customizing the products for each user.

In this paper, we present a value-based gamification framework in order to gamify daily activities. The central concept in our framework is values. A user's intrinsic motivation increases when he/she feels real or virtual goods or figures used in his/her activities valuable. Our framework introduces five values that are design tools to develop attractive gamification services. Then, we show how the values are used in Augmented Trading Card Game (Augmented TCG) [1] during its design as an example.

\section{Value-Based Gamification Design Framework}

This section briefly introduces the proposed five values, and presents a conceptual gamification framework to use these values.

The first value is the informative value. The value offers sufficient information to a user, and helps him/her to make a better decision. A typical example is an augmented 
reality service that superimposes useful information on an image captured by a camera. The service gives the user detailed information about the surrounding daily environment such as shopping information and route information. If a user does not find the necessary information, he/she may get lost and confused what to do, which might decrease his/her motivation to achieve his/her current goal.

The second value is the empathetic value. This value is achieved and enhanced by adding a virtual character or a pet to a service. For example, if a pretty virtual girl navigates a service, a typical male user usually feels the service more attractive. In a similar way, when a user takes care of a virtual pet in a service, he/she considers to continue using the service for a longer time. Especially, when a user maintains a long relationship with a virtual character or a pet, he/she could not give up using the service because he/she does not like to part from the character or the pet.

The third value is the persuasive value. The value gives a user feedback information according to his/her current situation. Also, the feedback information shows the future effect of a user's current activity. For example, a package of cigarettes shows photos of unhealthy lungs that are a result of long time smoking. Thus, while showing negative information might lead to a user stopping his/her current undesirable activities, showing positive information to a user would increase his/her motivation to engage into desirable activities.

The fourth value is the economic value. The value gives a sense of ownership to a user. Especially, physical tangibility is important to increase the sense of the ownership of an object. For example, people like to possess expensive jewelry or artworks. The rarity is a key to increase the sense of the ownership, and collecting rare objects increases the social status of a user as well. The sense of the ownership of an object is very important for a user to create his/her own original "empathetic story" with the object, which describes the user's feelings and attachment to the object and how the user's daily life has changed after he/she owns the object.

The fifth value is the ideological value. The value reminds a user important ideological concepts like friendship, justice and so on. However, the value is not explicitly presented to a user, but special stories carrying important ideological messages are used to explain the importance of such concepts implicitly. If a user knows the stories, then the characters appearing in the stories can be used as metaphors to show the ideological value.

Gamification offers the possibility to solve many real social problems. Current existing gamification frameworks take into account only game mechanics to motivate people and do not take into account the increase of the user's intrinsic motivation, which would lead to only partial solutions to the aimed social problems. A design with the proposed five values may enhance the current gamification frameworks and help people increase their self-efficacy that would make them believe that they are able to achieve their goals and to motivate them by themselves.

Fig. 1 shows how the five values are used to increase people's self-efficacy and to make them to think positively. The empathetic, economic and persuasive values offer people extrinsic incentives, and the informative value shows the reason to change human attitude or tips and tactics for making a better decision. Our framework is based on the transtheoretical model that is a psychological model to change human 
attitude. The four values are used as tools in the transtheoretical model to change a user's current behavior by reminding the importance and encourage his/her behavior change. On the other hand, the ideological value makes people's dream and expectation explicit to teach how changing a user's attitude realizes his/her dream. The combination of the extrinsic motivation and the ideological value enhances the intrinsic motivation and changes people's attitude, and makes people think more positively by increasing their self-efficacy. Therefore, the hurdles for people to solve some hard social problems become lower, and they become more confident and enthusiastic in taking an action to solve the problem through their increased self-efficacy and positive thinking.

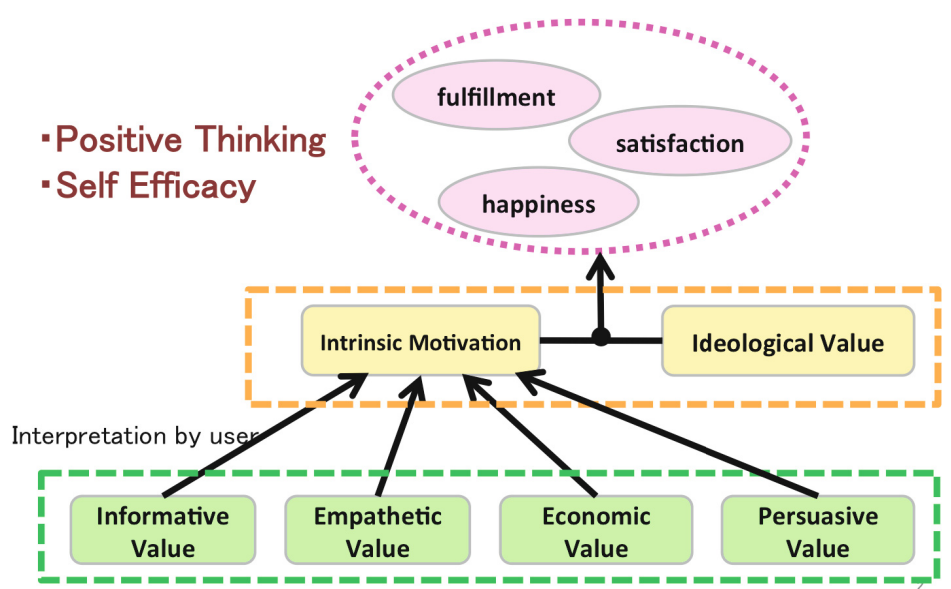

Fig. 1. Value-based Design Framework

\section{A Case Study: Augmented Trading Card Game}

Augmented TCG enhances the $\mathrm{Yu}$-Gi-Oh! trading card game (http://www.yugiohcard.com/en/) performed remotely by two persons. The two players are located in different places. Each player's cards in his/her battle field on the table in front of him/her are captured by a camera and projected on the opponent player's table. Also, each player is represented as the 3D model of a virtual character used in popular animations and games, and this character is shown to the opponent player. The virtual character is controlled using MS Kinect, its movement is synchronized with the movement and the behavior of the player it represents.

We briefly describe how we consider the presented five values while designing Augmented TCG as follows. The first is the informative value. Detailed information about the card that the opponent player currently holds in his/her hand is shown on a small display near a player. Especially, the information about the strength level of the card is hard to see during the game so showing the detailed information about the cards is useful to support the player's better decision making and strategy choice. The value encourages a player not to give up the game. 
The second is the empathetic value. In Augmented TCG, the virtual character representing players provokes their empathetic feeling and motivates them to play and enjoy the game better if that is one of their favorite characters. Currently, Link from The Legend of Zelda (http://zelda.com/) is chosen as a favorite character for players, but they can choose other favorite virtual characters for themselves as well. For some young players in Japan, favorite virtual characters are like close friends to them, so that they like to play with the character for a long time.

The third is the persuasive value. A small display shows a virtual character, that is illustrated on one of the cards in the player's deck, and encourages the player to win during the game. In TCG, trading cards are collected by each player with significant efforts. So, the player feels that his/her cards are very precious, and thus empathy with the characters depicted on the cards is easily initiated. Therefore, the encouragement by such character becomes a strong incentive to win the game.

The fourth is the economic value. In Augmented TCG, a player can use the physical paper cards from the TCG in the play. On the other hand, in the online version of TCG, cards are digitally represented in a virtual world. The player cannot touch the cards in the virtual world directly so he/she does not feel the strong sense of the ownership of cards. Using the physical paper cards is important to increase the sense of the ownership, and thus increases the motivation to collect more favorite cards to enjoy the game better. Especially, the rarity of the cards becomes a strong incentive to collect and own the favorite cards.

The last is the ideological value. Augmented TCG shows Yugi and Kaiba who are main heroes in the $\mathrm{Yu}-\mathrm{Gi}-\mathrm{Oh}$ ! animation story and opponent players. The characters represent some ideological concepts like friendship and justice in their background story. So, playing against Yugi and Kaiba reminds players the importance of such ideological factors because the players know the stories behind the characters. The extrinsic motivation established by the previous four values encourages enjoying a game, and the ideological value teaches a player the importance of friendship to really enjoy the game. The five values leads to the self-efficacy to improve a player's gaming skills with his/her friends' cooperation and support.

\section{$4 \quad$ Future Direction}

When using the transtheoretical model, growing intrinsic motivation is essential to raise a user's current stage. However, traditional approaches require a large amount of information that will make a user feel values to change his/her current attitude. We like to exploit the idea of using a character from a fictional animation or a game story, and thus by reminding the user the leitmotif of the character's story [1] to reduce the necessary information to grow his/her intrinsic motivation.

\section{Reference}

1. Sakamoto, M., Nakajima, T., Tokunaga, E., Alexandrova, T.: Augmenting Trading Card Play with Empathetic Virtual Characters. In: HumanCom 2012 (2012) 